Trauma Berufskrankh 2018 20 (Suppl4):S272-S278 https://doi.org/10.1007/s10039-018-0388-5 Online publiziert: 25. Juni 2018

(c) Springer Medizin Verlag $\mathrm{GmbH}$, ein Teil von Springer Nature 2018

CrossMark

\section{J. Geerling ${ }^{1}$ A. Ellwein ${ }^{1,2} \cdot$ H. Lill'}

${ }^{1}$ Klinik für Orthopädie und Unfallchirurgie, DIAKOVERE Friederikenstift, Hannover, Deutschland ${ }^{2}$ Department Schulter-, Knie- und Sportorthopädie, DIAKOVERE Annastift, Orthopädische Klinik, Medizinische Hochschule (MHH), Hannover, Deutschland

\title{
Möglichkeiten und Grenzen der konservativen Therapie: Fuß und Sprunggelenk
}

\section{Verletzungen im Bereich des Fußes und des Sprunggelenks können häufig konservativ behandelt werden. Hierbei heißt jedoch konservative Therapie nicht, dass keine Therapie durchgeführt werden soll, sondern eine an die jeweilige Verletzung angepasste Therapie mit entsprechenden Orthesen oder anderen Hilfsmitteln sowie die entsprechend adaptierte Physiotherapie.}

Im Folgenden soll hierbei auf Rupturen der Achillessehne, OSG(oberes Sprunggelenk)-Frakturen, Syndesmosenrupturen, OSG-Außenbandrupturen, funktionelle Instabilitäten und Mittelfußfrakturen des 5. Strahls eingegangen werden.

\section{Achillessehnenruptur}

Bei Achillessehnenrupturen ist bekannt, dass eine frühfunktionelle Behandlung in einer Orthese der Immobilisation in einem Gips überlegen ist. In einer Metaanalyse zeigten Kahn et al. [18] bei einer Analyse von 12 Artikeln mit insgesamt 800 Patienten, dass die Rerupturrate bei frühfunktioneller Behandlung auf 2,5-12,5\% gesenkt werden konnte im Gegensatz zur Behandlung im Gips mit Rerupturraten von $12,2-33 \%$.

Trotzdem ist die frühfunktionelle Nachbehandlung sowohl operativ als auch konservativ behandelter Rupturen doch noch nicht so weit verbreitet wie gedacht. In einer aktuellen Umfrage bei 213 Institutionen im deutschsprachigen
Raum fand Francewcyk [11] 243 Nachbehandlungsprotokolle, wobei hier auch teilweise zwischen der operativen und konservativen Therapie unterschieden wird. In allen Nachbehandlungsprotokollen fand sich diesbezüglich eine deutliche Restriktion der Belastung mit bis zu $40 \%$ Teilbelastung bis zur 6 . Woche.

Bei der Diagnose einer Achillessehnenruptur besteht noch weiterhin klinische Unsicherheit, sodass hier häufig noch eine Magnetresonanztomographie (MRT) zur Diagnostik durchgeführt wird. Garras [13] zeigte jedoch in einer Studie, dass allein durch eine korrekte klinische Untersuchung die Diagnose einer Achillessehnenruptur zu stellen ist. Sind nämlich der Thompson-Test und der Mates-Test pathologisch und lässt sich eine tastbare Delle fühlen, so fand sich intraoperativ immer eine komplette Achillessehnenruptur. Hingegen zeigte die MRT in diesen operierten Fällen nur eine komplette Ruptur bei $90 \%$ der Patienten.

Im Rahmen der klinischen Untersuchung sollte immer eine sonographische Kontrolle durchgeführt werden, da hierdurch auch das weitere Procedere festgelegt werden kann.

Eine konservative Therapie der Achillessehnenruptur ist dann indiziert, wenn unter Neutralstellung des oberen Sprunggelenks die Rupturenden nicht mehr als $10 \mathrm{~mm}$ Dehiszenz haben und unter $20^{\circ}$ Plantarflexion eine Adaptation erfolgt. Darüber hinaus kann mit der Sonographie ein mögliches Repositionshindernis, wie z. B. ein Rupturhämatom, ausgeschlossen werden. Eine weitere Voraussetzung der konservativen Therapie ist die Compliance des Patienten, da dieser die Orthese für 6 Wochen Tag und Nacht tragen muss. Aus der Orthese heraus ist eine entsprechende Körperpflege dann nur in Spitzfußstellung möglich.

Eine sonographische Kontrolle sollte zumindest im Intervall von 4 Wochen bis zum 3. Monat erfolgen. Die Behandlung erfolgt über 6 Wochen in einer Orthese mit einzustellender Spitzfußstellung z. B. $30 / 15 / 0^{\circ}$ über je 2 Wochen, anschließend noch Protektion im Sinne einer Fersenkeilerhöhung für weitere 4 Wochen [15].

Hiermit können adäquate Ergebnisse erzielt werden. In einer Übersicht aus Neumayer [20] zeigten konservativ behandelte Achillessehnenrupturen einen modifizierten Leppilathi-Score um 60 (maximal mögliche 70) Punkte. Allerdings zeigt sich die Rückkehr $\mathrm{zu}$ vorherigen Sportarten mit 37-75\% deutlich eingeschränkt.

Auch Lantto [19] fand in einer vergleichenden Studie zwischen konservativer und offen operativer Behandlung bei gleicher Nachbehandlung in einer Orthese unter primärer Vollbelastung nach 18 Monaten Follow-up keine signifikanten Unterschiede beider Gruppen in entsprechenden Follow-up-Scores. Allerdings zeigte sich eine deutlich bessere Muskelkraft für die Plantarflexion (bis $18 \%$ ) bei den operativ behandelten Patienten.

In einer aktuellen Zusammenfassung von Metaanalysen zu dieser Thematik von Braunstein et al. [4] fand sich beim Vergleich operativer zu konservativer 

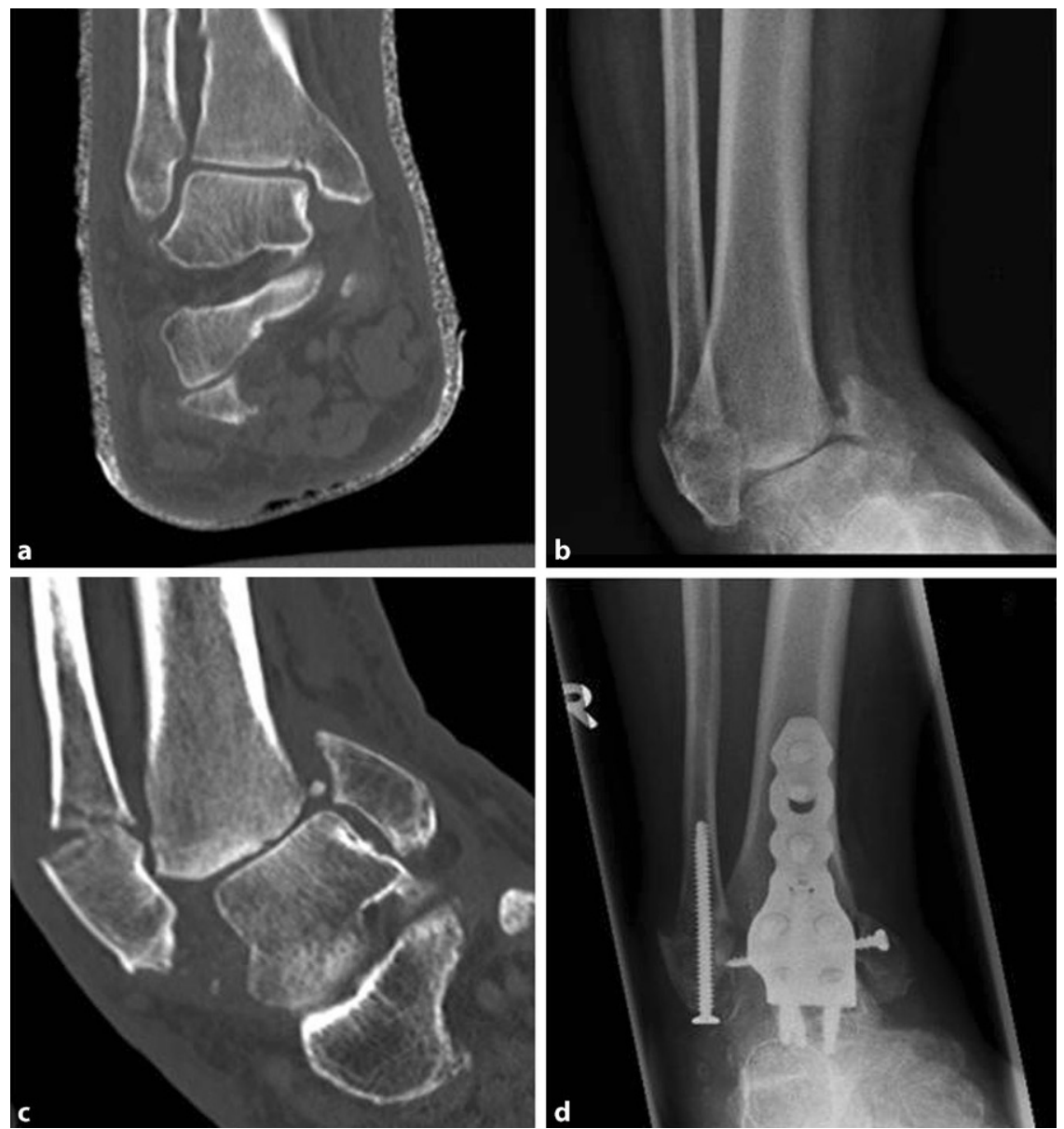

Abb. 1 ॥ 65-jährige Patientin mit konservativ behandelter instabiler OSG(oberes Sprunggelenk)-Fraktur. Bei Dislokation im Verlauf nach 3 Monaten OSG-Arthrodese (Einzelheiten s. Text). a Repositionskontrolle im Gips. b Röntgenkontrolle nach 2 Monaten. c CT Kontrolle nach 2 Monaten. d Behandlungsergebnis mittels OSG Arthrodese

Therapie ein Vorteil zugunsten der operativen Therapie im Hinblick auf eine höhere Funktion, geringere Rerupturrate und schnellere Rückkehr zur Arbeit allerdings dies zulasten der operativen Risiken wie Wundheilungsstörungen und insbesondere N.-suralis-Affektionen. Anzumerken ist, dass im Rahmen der entsprechenden Metaanalysen nur ein geringer Anteil an minimalinvasiven/perkutanen Operationsverfahren inkludiert war.

Im Vergleich dieser zu offenen Verfahren fand sich in der Analyse ein Vorteil der minimalinvasiven/perkutanen Verfahren - dies insbesondere mit Blick auf eine höhere Patientenzufriedenheit, schnellere Rückkehr zur Arbeit, geringere postoperative Schmerzen und Wundheilungsstörungen. Auch die reine Operationszeit war bei Anwendung der

\section{Unser Fazit}

Bei der Aufklärung über die konservative und operative Therapie werden diese Behandlungserfolge mit aufgeklärt. Insbesondere bei aktiveren Patienten führen wir diesbezüglich eine operative Therapie minimalinvasiv durch.

\section{Frakturen des oberen Sprunggelenks}

Bei OSG-Frakturen stellt sich die Frage, welche hiervon konservativ behandelt werden können und welche der operativen Versorgung zugeführt werden sollen.

Unverschobene oder minimal dislozierte Außenknöchelfrakturen des Typs Weber A/B können konservativ behandelt werden. Hierbei sollten jedoch Frakturen mit einer Verkürzung und einer Rotation detektiert und operativ versorgt werden. Ebenso können unverschobene Innenknöchelfrakturen, insbesondere mit horizontaler Frakturlinie, konservativ versorgt werden. Dies gilt besonders auch bei entsprechenden lokalen Kontraindikationen wie Ulzera, Infektionen im Fuß- oder Sprunggelenkbereich und Durchblutungsstörungen mit Gefahr einer Wundheilungsstörung.

Diese Frakturen können dann frühfunktionell in einer Orthese oder Außenknöchelschiene behandelt werden. In der frühen Phase sollte eine engmaschige Röntgenkontrolle erfolgen, um mögliche Dislokationen frühzeitig zu detektieren und ggf. doch noch eine operative Therapie frühzeitig, ohne entsprechende Notwendigkeit einer Korrekturosteotomie, durchzuführen.

Dietrich et al. [7] therapierten $38 \mathrm{~Pa}-$ tienten mit einer Weber-B-Fraktur bei maximaler Dislokation von $1 \mathrm{~mm}$ konservativ mit frühfunktioneller Nachbehandlung in einer Außenknöchelschiene. Hiermit erzielten 18 Patienten einen Oelerud-Molander-ankle-Score von 100 Punkten. Bei 4 Patienten zeigte sich in den Verlaufskontrollen eine Dislokation, sodass hier eine operative Therapie durchgeführt wurde.

Bei vertikalen Frakturverläufen im Innenknöchelbereich liegt jedoch eine instabile Situation vor. Bei möglichen Kon- 
traindikationen sollte eine engmaschige Kontrolle erfolgen.

Bei einer 65 jährigen Patientin mit Diabetes mellitus und einer noch nicht diagnostizierten Polyneuropathie erfolgte die initiale Behandlung einer Innenknöchelfraktur konservativ. Nach Anlage eines Repositionsgipses erfolgte die Durchführung einer CT-Untersuchung im ambulanten Bereich (• Abb. 1a).

Erst 2 Monate später erfolgte die Verlaufskontrolle, die eine deutliche Dislokation und sekundäre Außenknöchelfraktur zeigte (• Abb. 1b). In der dann durchgeführten Computertomographie(CT)-Diagnostik, auch zur Planung des weiteren Procedere, fand sich eine Impression im Tibiaplafond (- Abb. 1c), sodass hier nur die Durchführung einer OSG-Arthrodese erfolgen konnte (• Abb. 1d).

\section{Unser Fazit}

OSG-Frakturen werden in unserem Haus explizit analysiert und nur entsprechende Frakturen konservativ behandelt. Die Patienten werden nach Möglichkeit über unsere Ambulanz verlaufskontrolliert.

\section{Syndesmosenverletzungen}

Syndesmosenverletzungen treten im Zeitalter der häufig und schnell durchgeführten MRT-Diagnostik mehr und mehr in den Fokus.

Hierbei muss zunächst auf die Anatomie der Syndesmose eingegangen werden. Diese besteht aus 4 Anteilen, dem Lig. fibulotalare anterius, Lig. fibulotalare posterius, dem Lig. fibulotalare interosseum und der Membrana interossea. Diese erlauben eine dreidimensionale $\mathrm{Be}$ wegung der Fibula gegenüber der Tibia im Sinne einer Außenrotation von $2-5^{\circ}$ und einem posterioren Shift von 1-3 mm unter intakten Bedingungen. Das Lig. fibulotalare anterius dient als primärer $\mathrm{Au}$ ßenrotationsstabilisator, das Lig. fibulotalare posterius als primärer Stabilisator gegen den posterioren Shift. Die interossären Strukturen verhindern eine unphysiologische Translation.

Dieses ist für die Pathophysiologie entscheidend. Isolierte Syndesmosenverletzungen sind insgesamt selten, werden

Trauma Berufskrankh 2018 - 20 (Suppl 4):S272-S278

https://doi.org/10.1007/s10039-018-0388-5

(c) Springer Medizin Verlag GmbH, ein Teil von Springer Nature 2018

\section{J. Geerling · A. Ellwein $\cdot H$. Lill
Möglichkeiten und Grenzen der konservativen Therapie: Fuß und Sprunggelenk

Zusammenfassung

Verletzungen im Bereich des Fußes und des Sprunggelenks können häufig konservativ behandelt werden. Hierbei sollte jedoch eine entsprechend angepasste Therapie durchgeführt werden. Im Beitrag wird auf Verletzungen der Achillessehne, OSG(oberes Sprunggelenk)-Frakturen, Syndesmosenverletzungen, OSG-Außenbandrupturen sowie funktionelle Instabilitäten und Mittelfußfrakturen des 5 . Strahls eingegangen. Hierbei erfolgt ein aktueller Überblick über die konservative Therapie, allerdings werden auch entsprechende Grenzen aufgezeigt, wobei jeweils nicht auf eine entsprechende allumfassende Abhandlung eingegangen werden kann. Im Rahmen der entsprechenden konservativen Therapie sollte, soweit möglich, eine frühfunktionelle Therapie mit entsprechender Belastung und frühzeitiger Übungsbehandlung durchgeführt werden. Nicht nur dem Behandler, sondern auch dem Patienten sollte klar sein, dass die konservative Therapie häufig eine längere Rekonvaleszenzzeit als bei operativer Therapie mit sich bringt - dies im Gegensatz zur häufig schnelleren Behandlungszeit bei operativen Interventionen, aber mit dem Nachteil der Operationsrisiken.

Schlüsselwörter

Achillessehnenruptur - Syndesmosenverletzung · Proximale Mittelfußfrakturen . Verletzungen · Rekonvaleszenzzeit

\section{Possibilities and limits of conservative treatment: foot and ankle}

\section{Abstract}

Injuries of the foot and ankle can often be conservatively treated; however, an appropriately adjusted treatment should be performed. In this article injuries of the Achilles tendon, ankle fractures, injuries of the syndesmosis, lateral ankle ligament ruptures as well as ongoing functional instability and midfoot fractures of the fifth metatarsal are discussed. A current overview of the conservative therapy is given and the limits of this treatment are demonstrated; however, an all-inclusive discourse is not possible within the scope of this article. Within an appropriate conservative treatment, the focus should, as far as possible, be on early functional treatment with appropriate weight bearing and early exercise treatment. Not only the therapist but also the patient should be aware of the often protracted convalescence time compared to operative treatment. The shorter treatment times for surgical interventions are contrasted by the disadvantage of surgical risks.

\section{Keywords}

Achilles tendon rupture - Syndesmosis injury . Proximal metatarsal fractures - Injuries . Convalescence time in der Literatur mit 1-11\% der OSGVerletzungen angegeben. Als Unfallmechanismus ist ein Außenrotationstrauma des Fußes mit exzessiver Dorsalextension im Sprunggelenk zu sehen.

In Abhängigkeit der Verletzungsschwere erfolgt die Einteilung in 3 Grade [27].

- Grad 1 steht für eine stabile Situation, bei der es zu einer Partialruptur des Lig. fibulotalare anterius gekommen ist.

- Grad 2 steht für eine komplette Ruptur des Lig. fibulotalare anterius und partiale Ruptur der Membrana intraossea. Die Schwierigkeit bei dieser Situation besteht darin, dass die Verletzung stabil oder instabil sein kann.

- Grad 3 steht für eine komplette Läsion aller Anteile der Syndesmose mit oder ohne Deltabandbeteiligung und einer deutlichen Instabilität.

Hieraus ergeben sich entsprechende Therapieoptionen. Instabile Situationen Grad 3 müssen operativ versorgt werden.

Syndesmosenläsionen Grad 1 können, da stabil, konservativ therapiert werden. Unterstützt wird dies durch entsprechen- 
de biomechanische Untersuchungen von Candal-Couto et al. [6], die zeigten, dass bei einer Durchtrennung des Lig. fibulotalare anterius eine Aufweitung und ein posteriorer Shift der Syndesmose bzw. Fibula von maximal bis zu $1 \mathrm{~mm}$ auftreten.

Durch eine entsprechende Orthese oder Walker sollte eine Außenrotation in den ersten 6 Wochen nach der Verletzung vermieden werden. Allerdings muss sowohl dem Patienten als auch dem Behandler bewusst sein, dass diese Verletzung mit einer längeren Schmerzphase und Belastungsinsuffizienz einhergeht als Außenbandläsionen. Das „return-tosports“ kann bis zu doppelt so lange benötigen wie bei einer Außenbandläsion [14, 28].

Sowohl diagnostische als auch therapeutische Schwierigkeiten bestehen weiterhin bei Grad-2-Läsionen. Diese sind insgesamt nicht exakt definiert, es bestehen Übergänge zwischen stabilen und instabilen Läsionen. Bei entsprechender Klinik mit Verdacht auf eine Läsion sollte eine erweiterte Diagnostik über die MRT hinausgehend erfolgen. Es können Stressaufnahmen, möglicherweise auch im Seitenvergleich, durchgeführt werden.

Bei der MRT-Untersuchung sollten die axialen Schichten bis zu $6 \mathrm{~cm}$ oberhalb des OSG zur Verfügung stehen. In einer aktuellen Arbeit wird der sog. „broken ring of fire" beschrieben [5]. Dieser stellt ein halbmondförmiges Ödem $4-6 \mathrm{~cm}$ oberhalb des OSG dar, das um die Tibia liegt. Dieses Zeichen hat zwar nur eine Sensitivität von $49 \%$, aber eine Spezifität von $98 \%$. Dies impliziert bei Vorhandensein eine entsprechende Syndesmosenläsion.

Aber auch dies ist nur eine statische Aufnahme. Bei weiterem Verdacht auf eine instabile Situation sollte durchaus eine Arthroskopie des oberen Sprunggelenks mit dem Patienten besprochen werden. Hier kann die Stabilität bzw. Instabilität direkt unter Sicht verifiziert und in gleicher Operation therapiert werden. Ein intraoperatives Zeichen für eine Instabilität stellt die Möglichkeit des Eingehens und Drehens eines Tasthakens in der Syndesmose dar [26]. Auch ein Aufweiten des medialen oder lateralen Gelenkspalts zeigt eine solche Instabilität.

\section{Unser Fazit}

Entsprechende Verletzungen werden in unserem Haus unter Bildwandlerkontrolle untersucht. Bei entsprechender Klinik und fortbestehendem Verdacht auf eine Instabilität wird die Indikation zur Arthroskopie großzügig gestellt.

\section{Bandrupturen}

OSG-Außenbandrupturen sind die häufigste Verletzung des Bewegungsapparates [8]. Bei diesen Verletzungen ist bekannt, dass die nichtoperative frühfunktionelle Behandlung zu guten Ergebnissen führt. Diese besteht in der Therapie mit einer entsprechenden Außenknöchelschiene, welche die ersten 6 Wochen nach dem Trauma getragen werden sollte. Eine schmerzadaptierte Vollbelastung sollte durchgeführt werden.

Durch diese Therapie sind im Vergleich zur Immobilisation eine schnellere Rückkehr zu Arbeit und Sport, geringere Schwellneigung bei weniger Instabilität und eine bessere Beweglichkeit des OSG zu verzeichnen [9]. Ebenso sind Komplikationen wie tiefe Venenthrombosen geringer.

Auch im Vergleich zur direkten operativen Therapie sind die Ergebnisse vergleichbar, ohne dabei die Operationsrisiken zu haben [24].

Allerdings muss bei der frühfunktionellen Therapie bedacht werden, dass ca. $20-40 \%$ der Patienten Restbeschwerden oder gar Restinstabilitäten behalten. Auch können initial nach Abnahme der Orthese und Wiederaufnahme der Alltags- und Sportaktivitäten eine entsprechende Schwellneigung und Schmerzen bestehen, die sich im Laufe des ersten Jahres auf 5-10\% reduzieren [1]. Darüber hinaus ist eine persistierende Instabilität eine mögliche Ursache für eine Instabilitätsarthrose, die sich im Verlauf bei nicht therapierter Instabilität entwickeln kann.

Diesbezüglich sollte bei Patienten mit Beschwerden im Verlauf eine Unterscheidung zwischen mechanischer und funktioneller Instabilität erfolgen [12]. Eine mechanische Instabilität besteht in einer nachweisbaren pathologischen Instabilität des Gelenkes mit entsprechenden
Synovitiden und meist auch einer eingeschränkten Beweglichkeit.

Die funktionelle Instabilität beruht auf propriozeptiven Defekten und einer peronealen Schwäche mit Ermüdungserscheinungen und Giving-way-Episoden nach entsprechender Vorbelastung.

Bei diesen Patienten sollte ein intensives Propriozeptionstraining mit Koordinations- und Kraftübungen über etwa 3 Monate erfolgen. Auch die Protektion mit Orthesen bei sportlicher Belastung sollte durchgeführt werden. Hiermit können eine deutliche Besserung der subjektiven Beschwerden, eine verbesserte peroneale Reaktionszeit und eine reduzierte Rezidivrate an erneuten Umknicktraumen erzielt werden [25].

Bei keiner Besserung der Beschwerden sollte an ein Impingement mit intraartikulärer Narbenbildung gedacht werden, auch wenn dies nicht immer auf MRTs zu detektieren ist [17]. Die Gruppe um Kerr fand, dass bei 67 von 72 Patienten mit einer funktionellen Instabilität ein deutliches Narbengewebe intraartikulär $\mathrm{zu}$ verzeichnen war, insbesondere nach anterolateral. Durch das arthroskopische Débridement waren fast $75 \%$ der Patienten nach 6 Monaten Follow-up deutlich gebessert.

\section{Unser Fazit}

Bei Patienten mit entsprechender Klinik wird die Indikation zum arthroskopischen Débridement großzügig gestellt. Hier zeigt sich ebenfalls ein gutes postoperatives Ergebnis bei den Patienten (• Abb. 2).

\section{Mittelfußfrakturen des 5. Strahls}

Mittelfußfrakturen des 5. Strahls sind häufige Frakturen des Fußes. Hierbei sind Frakturen der Basis, des proximalen Schafts, der Schaftfraktur und Köpfchenfrakturen zu unterscheiden.

Proximale Frakturen der Zone I sind am häufigsten [16]. Zu den Frakturen des proximalen Schafts gibt es eine Vielzahl an Artikeln, auf die weiter unten eingegangen wird.

Insbesondere bei den Schaftfrakturen ist diese Anzahl deutlich geringer, sodass 

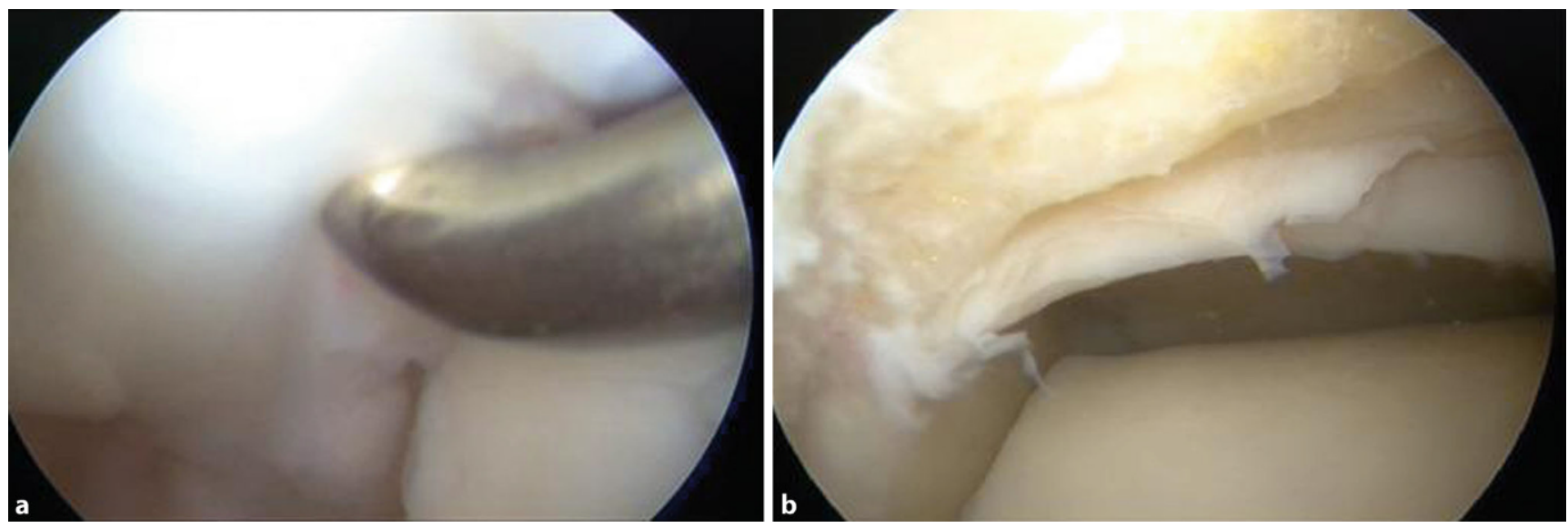

Abb. $2 \Delta$ a, b Patient mit funktioneller Instabilität. Intraoperativ findet sich ein anteriores Impingement (a). Nach entsprechendem Débridement deutliche Besserung der OSG(oberes Sprunggelenk)-Funktion (b)
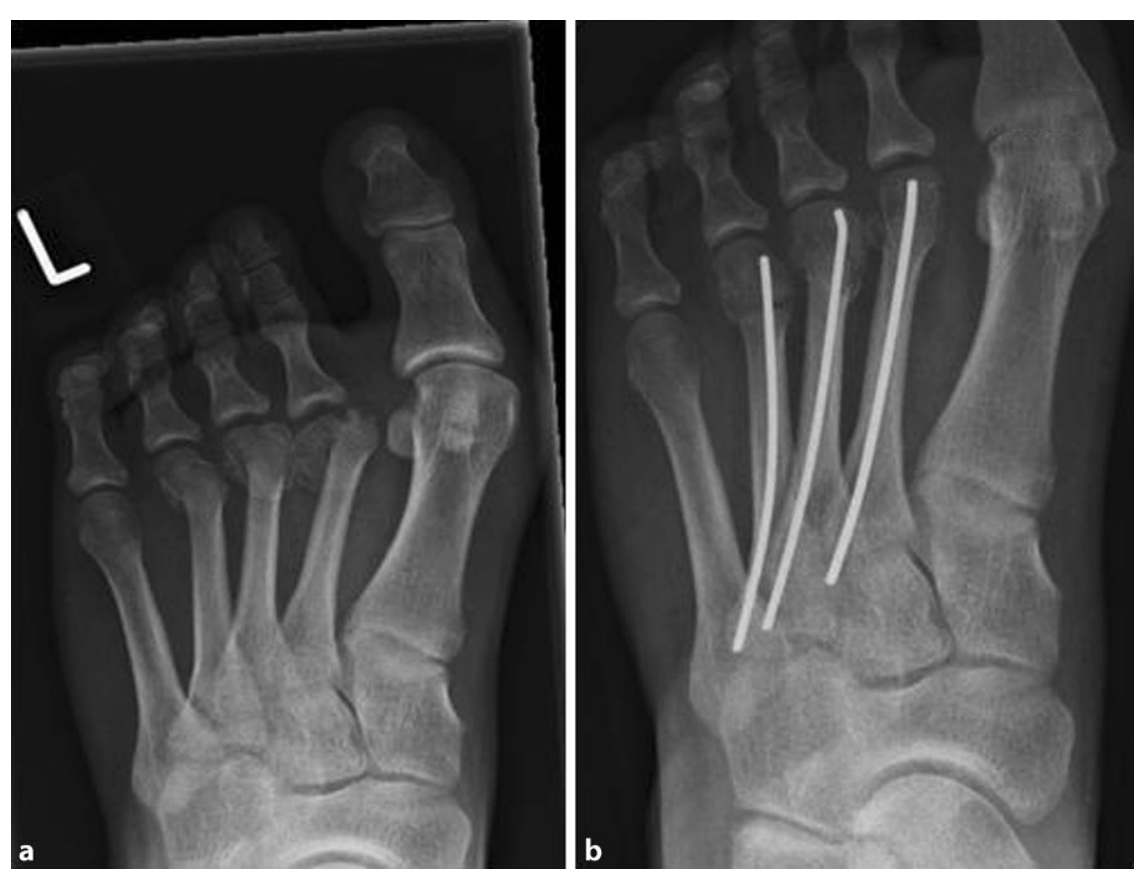

Abb. 3 × 21-jährige Patientin mit Kettenverletzung der Metatarsale-Köpfchen mit deutlicher Dislokation (a). Nach minimalinvasiver retrograder Schienung anatomische Reposition (b)

es hier wenig Evidenz zur konservativen oder operativen Therapie gibt.

Seit Sheref [22] gilt, dass bei Schaftfrakturen mit einer Dislokation von 3-4 $\mathrm{mm}$ und einer Achsabweichung von größer $10^{\circ}$ eine operative Therapie der Fraktur durchgeführt werden sollte.

In einer aktuellen Studie bei 142 Schaftfrakturen zeigten Aynardi et al. [2], dass diese jedoch mit einem guten Ergebnis frühfunktionell behandelt werden können. Die Nachbehandlung erfolgte im festen Schuh oder Verbandsschuh für 6 Wochen mit schmerzadaptierter Voll- belastung. Eingeschlossen wurden Frakturen mit weniger als $5 \mathrm{~mm}$ Dislokation. Es fand sich eine Pseudarthrosenrate von $2,1 \%$ bei einem „FAAM activities of daily living score" von 95,5 $( \pm 5,7)$ und einem „FAAM sports score“ von 92,7 $( \pm 9,1)$ bei maximal 100 Punkten nach einer Follow-up-Zeit von 3,5 Jahren.

Allerdings ist bei einer konservativen Therapie einschränkend anzugeben, dass die Heilungszeit bis zu 3,6 Monate betragen kann. Auch Bigsby et al. [3] fanden bei 39\% der Patienten mit einer konservativ behandelten Schaftfraktur noch deutliche Einschränkungen nach 4 Monaten.

Dahingegen fanden Thompson et al. bei 64 Patienten mit einer operativ versorgten Schaftfraktur eine Heilungszeit von 6,4 bis 8,6 Wochen bei nur einer „non-union“ und als weiterer Komplikation einem Infekt [23].

Bei konservativ behandelten Metatarsale-Frakturen sind auch mögliche Achsabweichungen nach dorsal oder plantar sowie Verkürzungen zu beachten, die möglicherweise im Verlauf zu entsprechenden Metatarsalgien führen können - dies insbesondere auch bei Metatarsale-Köpfchen-Frakturen, die bei entsprechender Abkippung zu diesen Problemen führen können.

Als Beispiel sei hier eine 21-jährige Patientin angeführt, die sich im Rahmen eines PKW-Unfalls eine Kettenverletzung der Metatarsale-Köpfchen zugezogen hatte. Es fanden sich eine deutliche Verkürzung und Plantardeviation (• Abb. 3). Durch eine antegrade intramedulläre K-Draht-Schienung, die minimalinvasiv über Stichinzisionen im Bereich der Metatarsale-Basen eingebracht wurde, konnte eine nahezu anatomische Reposition erzielt werden (• Abb. 4).

Bei proximalen Frakturen sind nach der Klassifikation von Lawrence und Botte die Avulsionsfraktur, die Jones-Fraktur und die diaphysäre Stressfraktur zu unterscheiden. Hierbei ist jedoch zu beachten, dass in vielen Publikationen die Jones-Fraktur im Sinne einer diaphysären Stressfraktur beschrieben wird und 


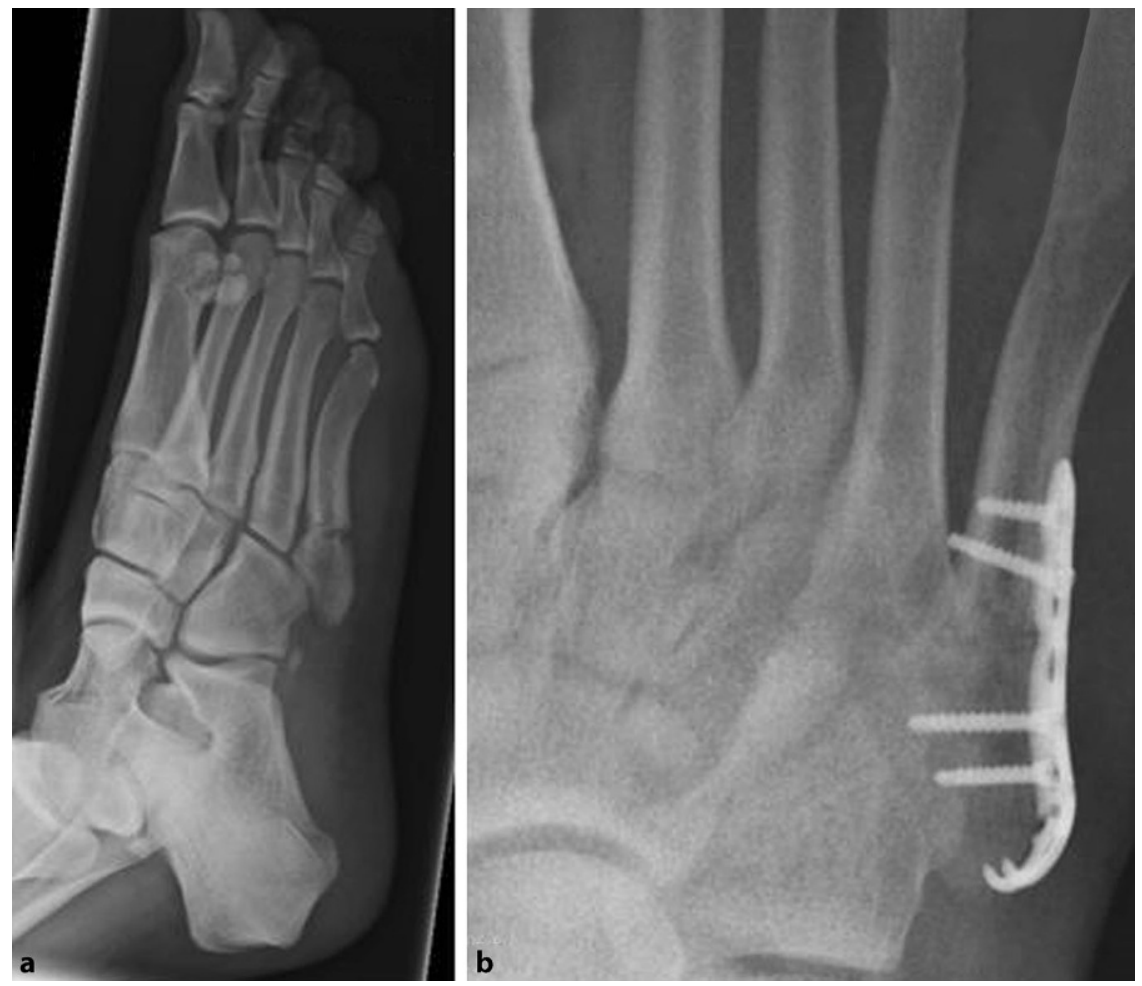

Abb. 4 ム Mittelfuß 5 Fraktur (MFK 5) diaphysäre Stressfraktur (a), versorgt mit Plattenosteosynthese (b)

somit die entsprechende Klassifikation in diesen Fällen nicht anwendbar ist.

Bezüglich der Behandlung zeigten Egol et al. [10] anhand von 50 eingeschlossenen Patienten, dass bei Frakturen der Zone 1 eine konservative Therapie zu guten Ergebnissen führen kann. Eingeschlossen wurden zur Hälfte intra- und extraartikuläre Frakturen, sowohl mit Frakturdislokationen kleiner und größer als $2 \mathrm{~mm}$. Die Behandlung erfolgte im festen Schuh und schmerzadaptierter Vollbelastung. Es zeigte sich nach $1 \mathrm{Jahr}$ eine Rückkehr zu dem Status vor der Verletzung von $100 \%$. Allerdings fand sich ein entsprechender Anteil von nur $20 \%$ nach 3 Monaten und $85 \%$ nach 6 Monaten. Die Frakturform und -dislokation hatten hierbei keinen Einfluss auf das Ergebnis, sodass die Autoren zu dem Schluss kamen, dass eine entsprechende konservative Therapie möglich ist, die Rekonvaleszenz aber 6 Monate oder länger dauern kann.

Wu et al. [29] zeigten im Vergleich von 21 operativ gegenüber 20 konservativ versorgten Avulsionsfrakturen, dass eine schnellere Rückkehr zur Vollbelastung und auch zur Arbeit bei operativ lyse ein schlechteres Ergebnis. Frakturen der Gruppe 3 zeigen unter konservativer Therapie, wie oben angegeben, eine signifikant schlechtere Heilungsrate und -zeit, sodass auch hier die operative Therapie bei entsprechenden Frakturlokalisationen empfohlen wird. Aufgrund dieser Analyse erfolgte durch die Gruppe die Einteilung in metaphysäre Frakturen (ehemals Zone 1 und 2) und in metadiaphysäre Frakturen (ehemals Zone 3).

\section{Unser Fazit}

Bei der Aufklärung über die konservative und operative Therapie werden diese Behandlungserfolge und Therapiedauern mit aufgeklärt. Insbesondere bei aktiveren Patienten führen wir diesbezüglich eher eine operative Therapie durch.

\section{Fazit für die Praxis}

- Bei Verletzungen im Fuß- und Sprunggelenkbereich sind häufig konservative Therapieformen möglich.

- Es sollte eine frühfunktionelle Therapie durchgeführt werden. Restriktive Behandlungsformen mit Ruhigstellung im Gips und restriktiver Belastung zeigen in den entsprechenden Analysen häufig ein schlechteres Outcome und eine verlängerte Behandlungszeit.

- Es sei auch noch mal auf die entsprechende Literatur hingewiesen, da dieser Übersichtsbeitrag teilweise nur den entsprechenden Einstand in das Thema bieten kann.

- Insgesamt bleibt festzuhalten, dass die Patienten nicht nur über Ergebnisse und Risiken einer entsprechenden Therapieform aufgeklärt werden sollten, sondern auch über entsprechende Behandlungszeiten.

\section{Korrespondenzadresse}

\section{Dr. J. Geerling}

Klinik für Orthopädie und Unfallchirurgie, DIAKOVERE Friederikenstift

Humboldtstr. 5, 30169 Hannover, Deutschland jens.geerling@diakovere.de 


\section{Einhaltung ethischer Richtlinien}

Interessenkonflikt. J. Geerling, A. Ellwein und H. Lill geben an, dass kein Interessenkonflikt besteht.

Dieser Beitrag beinhaltet keine von den Autoren durchgeführten Studien an Menschen oder Tieren.

The supplement containing this article is not sponsored by industry.

\section{Literatur}

1. Ardèvol J, Bolíbar I et al (2002) Treatment of complete rupture of the lateral ligaments of the ankle: a randomized clinical trial comparing cast immobilization with functional treatment. Knee Surg Sports Traumatol Arthrosc 10:371-377

2. Aynardi M, Pedowitz DI et al (2013) Outcome of nonoperative management of displaced oblique spiral fractures of the fifth metatarsal shaft. Foot Ankle Int 34:1619-1623

3. Bigsby E, Halliday Retal (2014) Functional outcome of fifth metatarsal fractures. Injury 45:2009-2012

4. Braunstein M, Baumbach SF et al (2017) Acute achilles tendon rupture : State of the art. Unfallchirurg 120:1007-1014

5. Calder J, Mitchell A et al (2017) The broken "ring of fire": a new radiological sign as predictor of syndesmosis injury? Orthop J Sports Med 5(3):2325967117695064

6. Candal-Couto JJ, Burrow D et al (2004) Instability of the tibio-fibular syndesmosis: have we been pulling in the wrong direction? Injury 35:814-818

7. Dietrich A, Lill Hetal (2002)Conservativefunctional treatment of ankle fractures. Arch Orthop Trauma Surg 122:165-168

8. DiGiovanni BF, Partal G et al (2004) Acute ankle injury and chronic lateral instability in the athlete. Clin Sports Med 23:1-19

9. van Dijk CN (2002) Management of the sprained ankle. Br JSports Med 36:83-84

10. Egol K, Walsh M et al (2007) Avulsion fractures of the fifth metatarsal base: a prospective outcome study. Foot Ankle Int 28:581-583

11. Frankewycz B, Krutsch Wetal (2017) Rehabilitation of Achilles tendon ruptures: is early functional rehabilitation daily routine? Arch Orthop Trauma Surg 137:333-340

12. Freeman MA (1965) Instability of the foot after injuries to the lateral ligament of the ankle. J Bone Joint Surg Br 47:669-677

13. Garras DN, Raikin SMetal (2012) MRl is unnecessary for diagnosing acute Achilles tendon ruptures: clinical diagnostic criteria. Clin Orthop Relat Res 470:2268-2273

14. Gerber et al (1998) Persistent disability associated with ankle sprains: a prospective examination of an athletic population. Foot Ankle 19:653-660

15. Hüfner T et al (2010) Konservativ-funktionelle Behandlung der Achillessehnenruptur. Unfallchirurg 113:699-704

16. Kane JM, Sandrowski K et al (2015) The epidemiology of fifth metatarsal fracture. Foot Ankle Spec 8:354-359

17. Kerr HL, BayleyEetal (2013) The role of arthroscopy in the treatment of functional instability of the ankle. Foot Ankle Surg 19:273-275

18. Khan RJ, Fick D et al (2005) Treatment of acute achilles tendon ruptures. A meta-analysis of randomized, controlled trials. JBone Joint Surg Am 87:2202-2210

19. Lantto I, Heikkinen J et al (2016) A prospective randomized trial comparing surgical and nonsurgical treatments of acute Achilles tendon ruptures. Am J Sports Med 44:2406-2414

20. Neumayer F, Mouhsine E et al (2010) A new conservative-dynamic treatment for the acute ruptured Achilles tendon. Arch Orthop Trauma Surg 130:363-368

21. Polzer H, Polzer S et al (2012) Acute fractures to the proximal fifth metatarsal bone: development of classification and treatment recommendations based on the current evidence. Injury 43:1626-1632

22. Shereff MJ (1990) Complex fractures of the metatarsals. Orthopedics 13:875-882

23. Thompson P, Patel V et al (2017) Surgical management of fifth metatarsal diaphyseal fractures: a retrospective outcomes study. J Foot AnkleSurg 56:463-467

24. Valderrabano V, Hintermann B et al (2006) Ligamentous posttraumatic ankle osteoarthritis. Am J Sports Med 34:612-620

25. Verhagen $E$, van der BeekA et al (2004) The effect of a proprioceptive balance board training program for the prevention of ankle sprains: a prospective controlled trial. Am J Sports Med 32:1385-1393

26. Wagner ML, Beumer A et al (2011) Chronic instability of the anterior tibiofibular syndesmosis of the ankle. Arthroscopic findings and results of anatomical reconstruction. BMC Musculoskelet Disord 12:212

27. Wolf BR et al (2002) Syndesmosis injuries in the athlete: when and how to operate. Curr Opin Orthop 13:151-154

28. Wright et al (2004) Ankle syndesmosis sprains in national hockey league players. Am J Sports Med 32(8):1941-1945. https://doi.org/10.1097/BLO. Ob013e31802eb471

29. Wu GB, Li B et al (2018) Comparative study of surgical and conservative treatments for fifth metatarsal base avulsion fractures (type I) in young adults or athletes. J Orthop Surg 26:2309499017747128

30. Yates J, Feeley I et al (2015) Jones fracture of the fifth metatarsal: Is operative intervention justified? A systematic review of the literature and metaanalysis of results. Foot (Edinb) 25:251-257 